Efimkina N. V., Maryin M. I., Tsvetkov V.L.

\title{
Personal predictors of information distortion
}

The digital world nowadays allows a quick access to any amount of information from different sources. Not only the speed of communication increases, but also the opportunities with the proper use of new technologies. Employees in high-risk professions also work with large amounts of information flows, and the extent to which they are able to perceive, evaluate and communicate information orally or in writing may influence the effective implementation of the assigned tasks.

In our research we have studied personal predictors of distortion of servicebased information in high-risk professions. At the first stage of the research, questionnaires were used for existing employees, which included questions aimed at establishing the presence of cases of distortion of service-based information in their activity; conditions and causes of this fact; questions of studying the impact of socio-psychological characteristics of employees, their needs, as well as managerial competemcies of supervisors on the distortion of service-based information.

At the second stage, with the help of the expert-sociometric program "Monitoring", 262 employees were evaluated, for each of which we obtained the indicators characterizing them in relation to distortion of service-based information, actions to ensure the reliability of service-based information, as well as personal attitudes and readiness to comply with the requirements of norms and rules of service behavior.

Taking into consideration the results obtained, persons prone to distortion of service-based information can be described as people seeking to avoid anxiety and fear by replacing the solution of subjectively complex tasks with simpler ones and, in so doing, converging to an early level of adaptive response. These employees tend to avoid actions that require a lot of tension, tend to solve their tasks with "someone else's hands", in case of omissions at work to designate the situation as a collective irresponsibility. The members of this group are oriented towards social approval and expect direct remuneration and satisfaction of personal needs. 